\title{
Downregulation of folate receptor $\alpha$ contributes to homocysteine-induced human umbilical vein endothelial cell injury via activation of endoplasmic reticulum stress
}

\author{
JIE CUI ${ }^{1,2}$, CHENG CAO $^{1}$, HUAN HU $^{1}$, HAO ZHANG $^{1}$, \\ YUJING YANG $^{1}$, LUQIAO WANG $^{1}$, XIAO HUANG $^{1}$ and PING LI ${ }^{1}$ \\ ${ }^{1}$ Department of Cardiovascular Medicine, The Second Affiliated Hospital of Nanchang University, Nanchang, Jiangxi 330006; \\ ${ }^{2}$ Department of Oncology, The First Affiliated Hospital of Anhui Medical University, Hefei, Anhui 230022, P.R. China
}

Received March 27, 2019; Accepted April 16, 2020

DOI: $10.3892 / \mathrm{mmr} .2020 .11204$

\begin{abstract}
Elevated plasma homocysteine (Hcy) levels are associated with endothelial dysfunction. Previous studies have indicated the role of endoplasmic reticulum (ER) stress in Hcy-induced endothelial injury. However, the mechanism of action remains unclear. In the current study, the role of human folate receptor $\alpha(\mathrm{hFR} \alpha)$ in Hcy-induced ER stress was investigated, along with endothelial injury in human umbilical vein endothelial cells (HUVECs). The results showed that a moderate dose of Hcy induced morphological injury and reduced viability of HUVECs. Furthermore, moderate-dose Hcy reduced hFR $\alpha$ expression in HUVECs. Notably, hFR $\alpha$ inhibition by RNA interference resulted in activation of the protein kinase RNA-like endoplasmic reticulum kinase pathway and increased apoptosis of HUVECs. In conclusion, this study demonstrated the presence of hFR $\alpha$ in HUVECs, and indicated its role in the regulation of ER stress and cell injury by Hcy.
\end{abstract}

\section{Introduction}

Homocysteine (Hcy) is a known risk factor for various cardiovascular diseases (CVDs) (1). It is well known that endothelial dysfunction plays a crucial role in CVDs $(2,3)$. Notably, Hcy is a modifiable risk factor for endothelial dysfunction. Evidence has demonstrated that hyperhomocysteinemia (HHcy), as defined as plasma total Hcy levels $\geq 15 \mu \mathrm{mol} / \mathrm{l}$ (1), is associated with impaired endothelium-dependent vascular dilation $(4,5)$.

Correspondence to: Professor Ping $\mathrm{Li}$, Department of Cardiovascular Medicine, The Second Affiliated Hospital of Nanchang University, 1 Minde Road, Nanchang, Jiangxi 330006, P.R. China

E-mail: lipingsydney@163.com

Key words: homocysteine, human umbilical vein endothelial cells, human folate receptor $\alpha$, endoplasmic reticulum stress, protein kinase RNA-like endoplasmic reticulum kinase
Previous studies reported that Hcy induced endoplasmic reticulum (ER) stress and apoptosis of human umbilical vein endothelial cells (HUVECs), indicating the involvement of ER stress in Hcy-induced endothelial injury (6-8). However, the precise mechanism of Hcy-induced endothelial dysfunction is not completely understood.

Hcy is formed during the conversion of methionine to cysteine (9). Folate plays an important role in Hcy catabolism via the remethylation pathways. Polymorphisms in the methylenetetrahydrofolate reductase gene or inadequate folate intake are associated with high Hcy levels and worse CVD outcomes (10-13). Moreover, human folate receptors (hFRs), particularly hFR $\alpha$, have a high affinity for folate and have a pivotal role in folate uptake (14). Previous studies have detected the expression levels of hFRs in healthy tissues, with higher levels of protein expression in human lung and kidney $(15,16)$. However, it remains unclear whether hFRs are expressed on HUVECs. Furthermore, little is known about the potential role of endothelial hFRs in Hcy-induced endothelial injury.

The present study investigated the role of hFRs in Hcy-induced HUVECs injury. Furthermore, the effect of hFR $\alpha$ inhibition through RNA interference (RNAi) on ER stress marker expression in HUVECs was studied.

\section{Materials and methods}

Materials. HUVECs (cat. no. KG419; http://www.keygentec. com.cn/index.php) were purchased from the Nanjing KeyGen Biotech Co., Ltd. Hcy was purchased from Sigma-Aldrich (Merck KGaA). Dulbecco's modified Eagle's medium (DMEM), fetal bovine serum (FBS) and penicillin/streptomycin were purchased from Gibco (Thermo Fisher Scientific, Inc.). TransLipid ${ }^{\circledR}$ HL Transfection Reagent and Cell Counting Kit-8 (CCK-8) were purchased from Beijing TransGen Biotech Co., Ltd. The bicinchoninic acid (BCA) protein assay kit was purchased from Wuhan Boster Biological Technology Ltd. Dimethyl sulfoxide, which was used for freezing and storing HUVECs, and radioimmunoprecipitation assay (RIPA) buffer were purchased from Applygen Technologies, Inc. The AnnexinV-fluorescein isothiocyanate (FITC) Apoptosis Detection kit was purchased from BD Pharmingen. The anti- 
body against hFR $\alpha$ (cat.no. ab3361) was purchased from Abcam. The antibody against $\beta$-tubulin (cat. no. sc-5274) was purchased from Santa Cruz Biotechnology, Inc. Antibodies against activating transcription factor 4 (ATF4; cat. no. 60035-1-lg) and caspase 12 (cat. no. 55238-1-AP) were purchased from Wuhan Sanying Biotechnology. Antibodies against protein kinase RNA-like ER kinase (PERK; cat. no. 5683), phosphorylated (p)-PERK (cat. no. 3179), p-eukaryotic translation initiation factor $2 \alpha$ (p-eIF2 $\alpha$; cat. no. 3398) and C/EBP homologous protein (CHOP; cat. no. 2895) were purchased from Cell Signaling Technology, Inc. The antibody against eIF2 $\alpha$ (cat. no. AF6087) was purchased from Affinity Biosciences, Inc. The antibody against $\beta$-actin (cat. no. TA-09) was purchased from OriGene Technologies, Inc. The western blotting detection reagents were purchased from Sigma-Aldrich (Merck $\mathrm{KGaA}$ ). The small interfering RNA (siRNA) targeting hFR $\alpha$ and control siRNA were purchased from Novobio Co., Ltd. The siRNA targeting PERK and corresponding control siRNA were purchased from Shanghai GenePharma Co., Ltd.

Cell culture and treatment. HUVECs were cultured in DMEM with high sugar, containing 10\% FBS and $1 \%$ penicillin/streptomycin at $37^{\circ} \mathrm{C}$ in a $5 \% \mathrm{CO}_{2}$ humidified atmosphere. The medium was changed every $48 \mathrm{~h}$, the cells were passaged every 2-3 days. For Hcy treatment, HUVECs were incubated with mild-to-moderate concentrations of Hcy $(0,50,100$ and $200 \mu \mathrm{M})$. For knocking down hFR $\alpha$, HUVECs were transfected with siRNA targeting hFR $\alpha$. The sequences of siRNAs were as follows: FR $\alpha$-siRNA-1 sense, 5'-GGACUGAGCUUCUCAAUGUTT-3' and anti-sense, 5'-ACAUUGAGAAGCUCAGUCCTT-3'; FR $\alpha$-siRNA-2 sense, 5'-GAUGUUUCCUACCUAUAUATT-3' and anti-sense, 5'-UAUAUAGGUAGGAAACAUCTT-3'; FR $\alpha$-siRNA-3 sense, 5'-CCACUGUUCUGUGCAAUGATT-3' and anti-sense, 5'-UCAUUGCACAGAACAGUGGTT-3'; and negative control siRNA sense, 5'-UUCUCCGAACGUGUCACGUTT-3' and anti-sense, 5'-ACGUGACACGUUCGGAGAATT-3'. For knocking down PERK, HUVECs were transfected with siRNAs targeting PERK. The sequences were as follows: PERKsiRNA-1 sense, 5'-ACCTCCAAGACCAACCACTTT-3' and anti-sense, 5'-AAAGTGGTTGGTCTTGGAGGT-3'; PERK-siRNA-2 sense, 5'-GUAGCUGGAAUGACAUAA ATT-3' and anti-sense, 5'-UUUAUGUCAUUCCAGCUA CTT-3'; PERK-siRNA-3 sense, 5'-GUGGAAAGGUGAGGU AUAUTT-3' and anti-sense, 5'-AUAUACCUCACCUUUCCA CTT-3'; and negative control siRNA sense, 5'-UUCUCC GAACGUGUCACGUTT-3' and anti-sense 5'-ACGUGACAC GUUCGGGATT -3'.

For cell transfection, HUVECs were plated in a 6-well plate, at a density of $\sim 50 \%$. The cells were transfected with $20 \mu \mathrm{M}$ corresponding siRNA or negative control siRNA using TransLipid ${ }^{\circledR}$ HL Transfection reagent (TransGen Biotech Co., Ltd.) and incubated at room temperature for $20 \mathrm{~min}$, according to the manufacture's protocol. After $48 \mathrm{~h}$, the effect of target gene knockdown was confirmed by western blotting.

Cell morphology, viability and apoptosis. For morphological observation, HUVECs were seeded into 6-well plates $\left(5 \times 10^{5}\right.$ cells/well) and incubated with different concentrations of Hcy $(0,50,100$ and $200 \mu \mathrm{M})$ for $24 \mathrm{~h}$ at $37^{\circ} \mathrm{C}$. Cell morphology was examined with an inverted light microscope Leica DMi1 (magnification, x50; Leica Microsystems GmbH).

CCK-8 assay was used to measure cell viability according to the manufacturer's protocol. Cells $\left(1 \times 10^{5}\right.$ cells $\left./ \mathrm{ml} ; 100 \mu \mathrm{l} / \mathrm{well}\right)$ were seeded in a 96-well culture plate and incubated for $24 \mathrm{~h}$. After pretreatment with Hcy at different concentrations $(0,50$, 100 and $200 \mu \mathrm{M})$ for $24 \mathrm{~h}, \mathrm{CCK}-8(10 \mu \mathrm{l} / 100 \mu \mathrm{l}$ fresh culture medium) was added to each well and incubated for $1 \mathrm{~h}$ at $37^{\circ} \mathrm{C}$. A microplate reader (Thermo Fisher Scientific Inc.) was used to measure the absorbance at a wavelength of $490 \mathrm{~nm}$. Cell viability $=\left(\mathrm{OD}_{\text {treatment }}-\mathrm{OD}_{\text {blank }}\right) /\left(\mathrm{OD}_{\text {control }}-\mathrm{OD}_{\text {blank }}\right) \times 100 \%$, where OD refers to optical density.

Annexin V-FITC/propidium iodide (PI) double staining was performed to measure cell apoptosis. After incubation with siRNA targeting $\mathrm{hFR} \alpha$ for $24 \mathrm{~h}$, the HUVECs were collected and centrifuged at $300 \mathrm{xg}$ for $10 \mathrm{~min}$ at $4^{\circ} \mathrm{C}$, washed three times with cold PBS and then resuspended in binding buffer $\left(1 \times 10^{6}\right.$ cells $\left./ \mathrm{ml}\right)$. Subsequently, cells were incubated with Annexin V-FITC for $15 \mathrm{~min}$ and then PI for $5 \mathrm{~min}$ at room temperature in the dark. The results were measured using a Backman CytoFLEX LX flow cytometer (Beckman Coulter, Inc.) and analyzed with CytExpert software (v2.1; Beckman Coulter, Inc.).

Reverse transcription-quantitative polymerase chain reaction $(R T-q P C R)$. The mRNA expression levels of $h F R \alpha$, hFR $\beta$ and hFR $\gamma$ were determined by RT-qPCR after $24 \mathrm{~h}$ of Hcy treatment. mRNA expression levels of solute carrier family 46 member 1 (SLC46A1) and solute carrier family 19 member 1 (SLC19A1), which are the main folate transporters in mammals $(17,18)$ were also determined. Briefly, total RNA was extracted from HUVECs using TRIzol ${ }^{\circledR}$ reagent (Invitrogen; Thermo Fisher Scientific, Inc) and reverse transcription was performed with a EasyScript ${ }^{\circledR}$ One-Step RT-PCR SuperMix kit (Beijing Transgen Biotech Co., Ltd.). For the synthesis of the first-strand cDNA, a total of $20 \mu \mathrm{l}$ reaction solution was incubated for $30 \mathrm{~min}$ at $45^{\circ} \mathrm{C}$. qPCR was performed using an ABI 7900 real-time PCR system (Applied Biosystems; Thermo Fisher Scientific, Inc.) with TransStart ${ }^{\circledR}$ Tip Green qPCR SuperMix kit (Beijing Transgen Biotech Co., Ltd.). For PCR amplification, a total of $20 \mu \mathrm{l}$ reaction solution, which included $2 \mu \mathrm{lcDNA}$, was first incubated for $30 \mathrm{sec}$ at $94^{\circ} \mathrm{C}$, followed by 42 amplification cycles (denaturation at $94^{\circ} \mathrm{C}$ for $5 \mathrm{sec}$, annealing at $55^{\circ} \mathrm{C}$ for $15 \mathrm{sec}$ and extension at $72^{\circ} \mathrm{C}$ for $10 \mathrm{sec}$ ). Fold changes in target gene expression were determined using the $2^{-\Delta \Delta \mathrm{Cq}}$ method (19) and relative levels of mRNA were normalized to mRNA levels of $\beta$-actin for each sample. The following primers for RT-qPCR were used: hFR $\alpha$ sense, 5'-GAATGCCTGCTGTTCTACCA-3' and antisense, 5'-TGCGACAATCTTCCCACC-3'; hFR $\beta$ sense, 5'-ATGCCACTTCTGCTGCTTCT-3' and antisense, 5'-AGTGACTCCAGAGGCCTTCA-3'; hFR $\gamma$ sense, 5'-TCAATGTCTGCATGAACGCCAAGC-3' and antisense, 5'-TAAAGTTGTACAGGCGGGAGGTGT-3';SLC46A1 sense, 5'-CTGGACCCTCTACATGAACG-3' and antisense, 5'-GGT AGAGTGAGTTGAAGATG-3'; SLC19A1 sense, 5'-CCTCGT GTGCTACCTTTGCTT-3' and antisense, 5'-TGATCT CGTTCGTGACCTGCT-3'; and $\beta$-actin sense, 5'-AGCGAG CATCCCCCAAAGTT-3' and antisense, 5'-GGGCAC GAAGGCTCATCATT-3'. 
A a

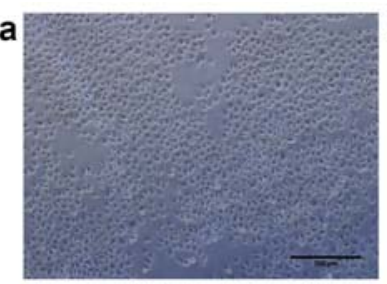

C

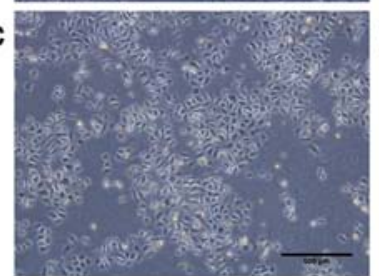

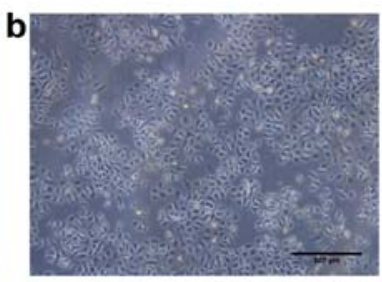

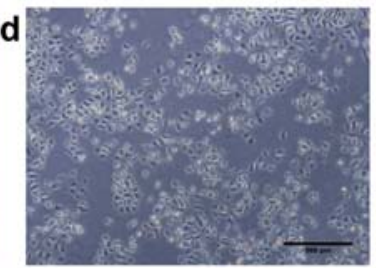

C
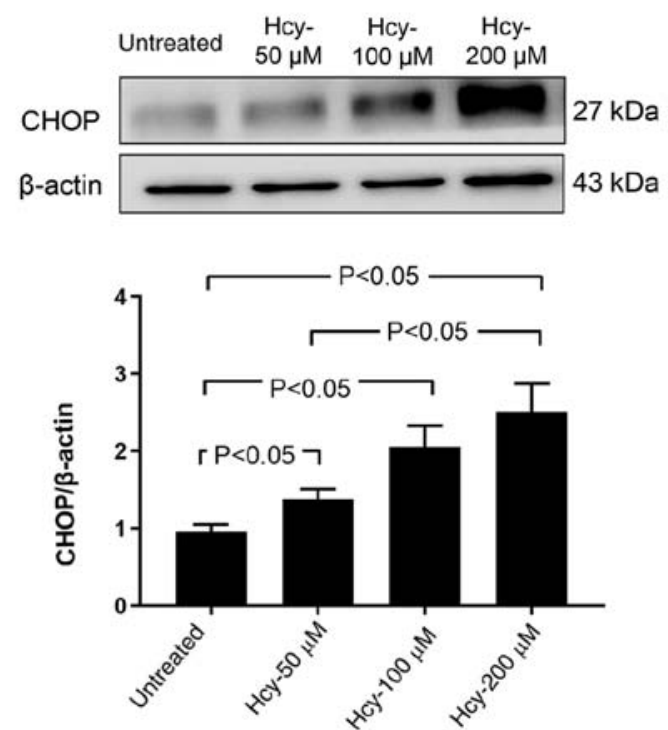

B

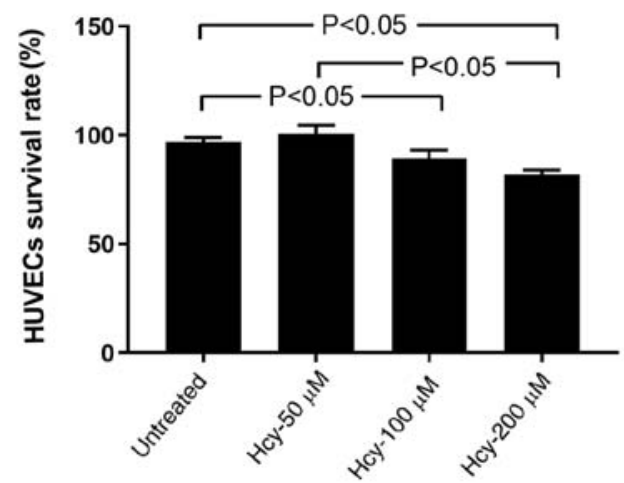

D
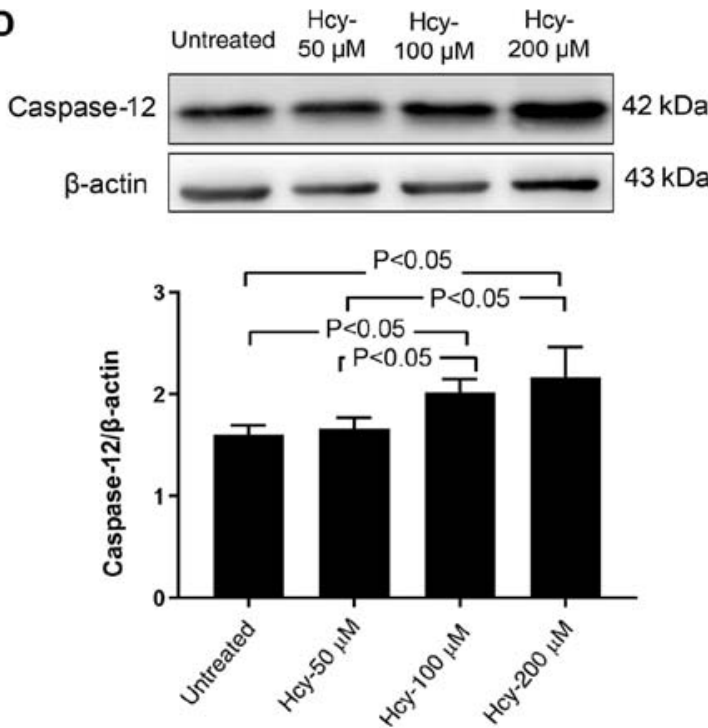

Figure 1. Effect of Hcy treatment on morphological changes, cell viability and apoptosis of HUVECs. (A) Representative images (magnification, x50) of HUVECs in the (a) untreated, (b) 50, (c) 100 and (d) $200 \mu \mathrm{M}$ Hcy groups. (B) Cell Counting kit-8 assays of cell viability. (C) Representative western blot of CHOP and semi-quantification of $\mathrm{CHOP} / \beta$-actin. (D) Representative western blot of caspase 12 and semi-quantification of caspase $12 / \beta$-actin. Data are presented as mean \pm standard error. Statistical analysis was conducted by one-way ANOVA followed by Tukey's multiple comparison post hoc test. HUVECs, human umbilical vein endothelial cells; Hcy, homocysteine, CHOP, C/EBP homologous protein.

Western blot analysis. For western blotting, HUVECs were collected and total proteins were lysed using RIPA buffer on ice; proteins were quantified by the BCA protein assay kit. Equal amounts of total protein $(50 \mu \mathrm{g})$ were separated by sodium-dodecyl sulfate polyacrylamide gel electrophoresis on a $10 \%$ gel, and transferred to polyvinylidene difluoride membranes. The membranes were blocked with $5 \%$ skimmed milk for $2 \mathrm{~h}$ at room temperature and then incubated overnight at $4^{\circ} \mathrm{C}$ with primary antibody against $\mathrm{hFR} \alpha(1: 800$ dilution), PERK (1:1,000 dilution), p-PERK (1:1,000 dilution), ATF4 (1:1,000 dilution), caspase 12 (1:1,000 dilution), CHOP (1:1,000 dilution), eIF2 $\alpha$ (1:400 dilution), p-eIF2 $\alpha$ (1:1,000 dilution), $\beta$-actin (1:2,000 dilution) or $\beta$-tubulin (1:1,000 dilution), followed by incubation with the respective HRP-conjugated secondary antibodies (cat. nos. HS101 and HS201; 1:5,000 dilution; Beijing TransGen Biotech Co., Ltd.) for $2 \mathrm{~h}$ at room temperature. Finally, the target bands were visualized using an enhanced chemiluminescence detection reagent (cat. no. DW101; Beijing Transgen Biotech Co., Ltd.) and images were captured by Quantity One 1-D analysis software (Bio-Rad Laboratories, Inc.). Intensity of the bands were assessed with ImageJ (v1.46, National Institutes of Health) and normalized to the intensity of loading controls $\beta$-actin or $\beta$-tubulin.

Statistical analysis. The experiments were repeated three times and the data are expressed as mean \pm standard error. Statistical analysis was performed with the SPSS statistics program (v22.0; IBM Corp.). One-way ANOVA followed by Tukey post hoc test or Kruskal-Wallis followed by Dunn-Bonferroni post hoc test were applied as appropriate. A two-sided $\mathrm{P}<0.05$ was considered to indicate a statistically significant difference.

\section{Results}

Hcy induces morphological changes and decreases viability of HUVECs. As shown in Fig. 1A, HUVECs treated with 0 and $50 \mu \mathrm{M}$ Hcy were smooth and plump, arranged in a tight and neat conformation. Conversely, exposure of HUVECs to higher concentrations of Hcy (100 and $200 \mu \mathrm{M})$ induced marked changes. There were fewer adherent cells, and an increase in cell shedding (Fig. 1A). 
A

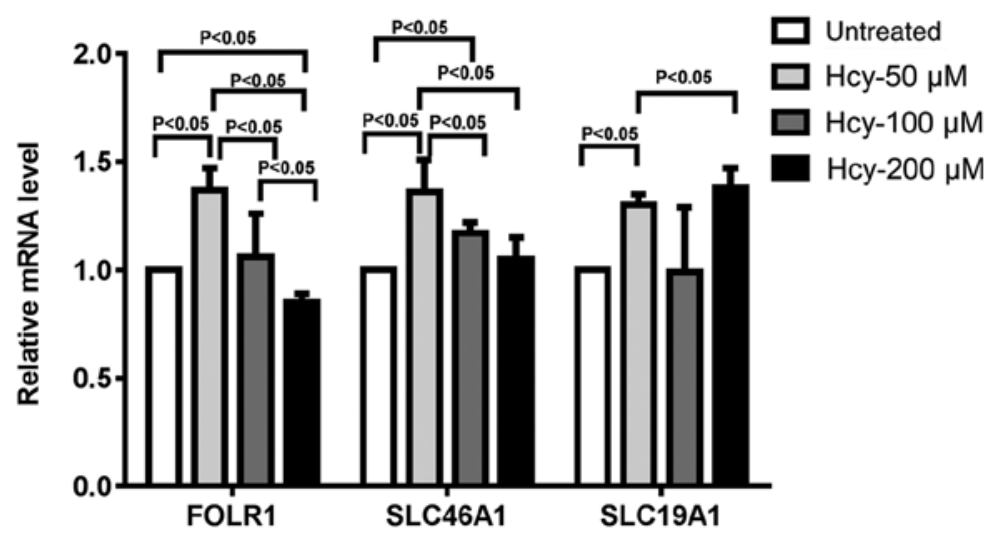

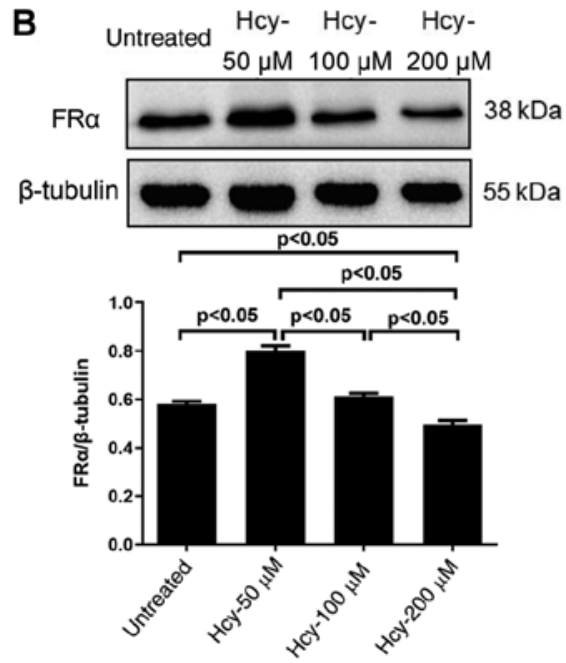

Figure 2. Effect of Hcy treatment on hFR $\alpha$ expression. (A) mRNA expression levels of FOLR1 (hFR $\alpha$ ), SLC46A1 and SLC19A1. (B) Representative western blot of $h F R \alpha$ and semi-quantification of hFR $\alpha / \beta$-tubulin. Data are presented as mean \pm standard error. (A) Statistical analysis was conducted by Kruskal-Wallis test followed by Dunn-Bonferroni post-hoc test between each group. (B) Statistical analysis was conducted by one-way ANOVA followed by Tukey's multiple comparison post hoc test. hFR $\alpha$, human folate receptor $\alpha$; Hcy, homocysteine; SLC46A1, solute carrier family 46 member 1; SLC19A1, solute carrier family 19 member 1.

As shown in Fig. 1B, no significant differences in cell viability were found between untreated cells and cells treated with $50 \mu \mathrm{M}$ Hcy $(\mathrm{P}>0.05)$. Hcy at a concentration of 100 and $200 \mu \mathrm{M}$ significantly reduced the percentage of viable cells compared with untreated cells $(\mathrm{P}<0.05)$. Cells treated with $200 \mu \mathrm{M}$ Hcy were also significantly less viable than cells treated with $50 \mu \mathrm{M}$ Hcy $(\mathrm{P}<0.05$; Fig. $1 \mathrm{~B})$.

As shown in Fig. 1C, Hcy at 50, 100 and $200 \mu \mathrm{M}$ significantly increased the protein expression of $\mathrm{CHOP}$, relative to $\beta$-actin, compared with the untreated cells $(\mathrm{P}<0.05)$. As shown in Fig. 1D, treatment with Hcy at 100 and $200 \mu \mathrm{M}$ significantly increased the protein expression of caspase 12 , relative to $\beta$-actin, compared with the untreated cells and cells treated with $50 \mu \mathrm{M}$ Hcy $(\mathrm{P}<0.05)$.

Hcy reduces expression of $m R N A$ and protein expression levels of $h F R \alpha$. As shown in Fig. 2A, treatment with $50 \mu \mathrm{M}$ Hcy for $24 \mathrm{~h}$ significantly increased the mRNA expression of hFR $\alpha$ (FOLR1), SLC46A1 and SLC19A1 (P<0.05) compared with the untreated cells. The mRNA expression of hFR $\alpha$ was significantly lower in cells treated with $200 \mu \mathrm{M}$ Hcy compared with untreated cells and cells treated with 50 and $100 \mu \mathrm{M} \mathrm{Hcy}$ $(\mathrm{P}<0.05$; Fig. 2A). Compared with cells treated with $50 \mu \mathrm{M}$ Hcy, the mRNA expression levels of hFR $\alpha$ were significantly lower in cells treated with 100 and $200 \mu \mathrm{M}$ Hcy $(\mathrm{P}<0.05)$. Cells treated with $50 \mu \mathrm{M}$ Hcy also expressed significantly higher levels of SLC46A1 mRNA compared with cells treated with 100 and $200 \mu \mathrm{M}(\mathrm{P}<0.05)$. Conversely, the mRNA levels of SLC19A1 increased significantly in cells treated with $200 \mu \mathrm{M}$ Hcy, compared with cells treated with $50 \mu \mathrm{M}$ Hcy $(\mathrm{P}<0.05$; Fig. 2A). On the other hand, the study failed to detect the mRNA levels of hFR $\beta$ and hFR $\gamma$ (data not shown).

The protein expression levels of $h F R \alpha$ in HUVECs were also measured after Hcy treatment for $48 \mathrm{~h}$. As shown in Fig. 2B, the protein expression of $\mathrm{hFR} \alpha$ was significantly increased in cells treated with $50 \mu \mathrm{M}$ Hcy compared with untreated cells $(\mathrm{P}<0.05)$. Compared with cells treated with $50 \mu \mathrm{M}$ Hcy, protein expression levels in cells treated with $100 \mu \mathrm{M}$ Hcy were signifi- cantly reduced $(\mathrm{P}<0.05)$. Compared with the untreated cells, or cells treated with 50 or $100 \mu \mathrm{M}$ Hcy, cells treated with $200 \mu \mathrm{M}$ Hcy also had a significantly lower protein expression $(\mathrm{P}<0.05)$.

hFRa knockdown increases apoptosis and induces PERK activation in HUVECs. To determine the role of hFR $\alpha$ in Hcy-induced HUVECs injury, hFR $\alpha$ expression was inhibited using siRNA. Transfection was confirmed by immunofluorescence staining with siRNAs that had an immunofluorescent component (data not shown). hFR $\alpha$ expression was significantly reduced in HUVECs transfected with hFR $\alpha$ siRNA compared with HUVECs that were not transfected (untreated) or transfected with a non-specific control siRNA (Fig. 3A and B). Among the three siRNAs, hFR $\alpha$-siRNA-1 generated the most significant knockdown results and was therefore chosen for further experiments. Flow cytometric analysis was conducted $12 \mathrm{~h}$ post-siRNA transfection. As shown in Fig. 3C and D, the apoptotic rate of HUVECs transfected with $\mathrm{hFR} \alpha$ siRNA was significantly higher compared with the untreated and the control siRNA groups $(\mathrm{P}<0.05)$.

The PERK signaling pathway, which is a sensor of the unfolded protein response in ER stress (20), was also analyzed. As shown in Fig. 3E and F, the p-PERK/PERK ratio was significantly higher in cells transfected with $h F R \alpha$ siRNA than the untreated and control siRNA groups $(\mathrm{P}<0.05)$. In addition, as shown in Fig. $3 \mathrm{E}, \mathrm{G}, \mathrm{H}$ and $\mathrm{I}, \mathrm{hFR} \alpha$ siRNA transfection caused a significant increase in the expression of ATF4 mRNA $(\mathrm{P}<0.05)$ and $\mathrm{p}$-eIF $2 \alpha(\mathrm{P}<0.05)$ in comparison with the untreated and control siRNA groups.

Knockdown of PERK attenuates Hcy-induced cell injury in HUVECs. As shown in Fig. 4A and B, in cells treated with 100 and $200 \mu \mathrm{M}$ Hcy for $48 \mathrm{~h}$ the $\mathrm{p}-\mathrm{PERK} / \mathrm{PERK}$ ratio was significantly increased compared with untreated cells $(\mathrm{P}<0.05)$. In order to determine the role of PERK in Hcy-induced injury, PERK mRNA expression was knocked down using siRNA (Fig. 4C and D). Among the three siRNAs, PERK-siRNA-1 generated the most significant knockdown results and 
A

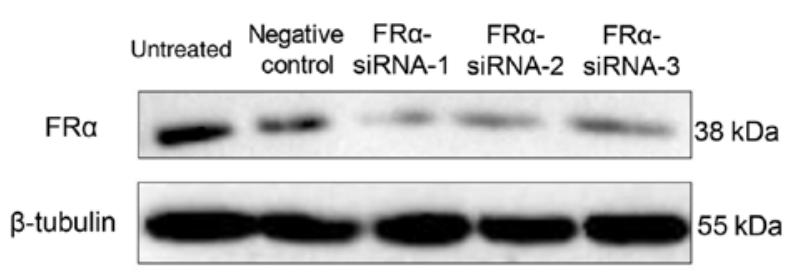

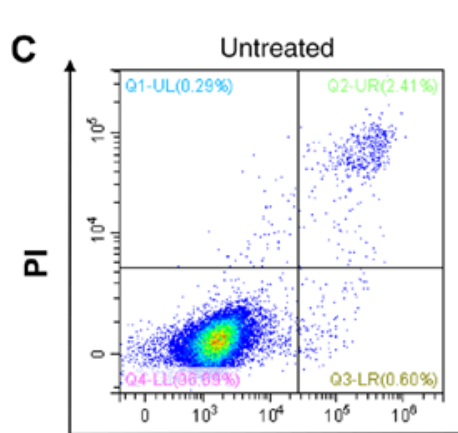
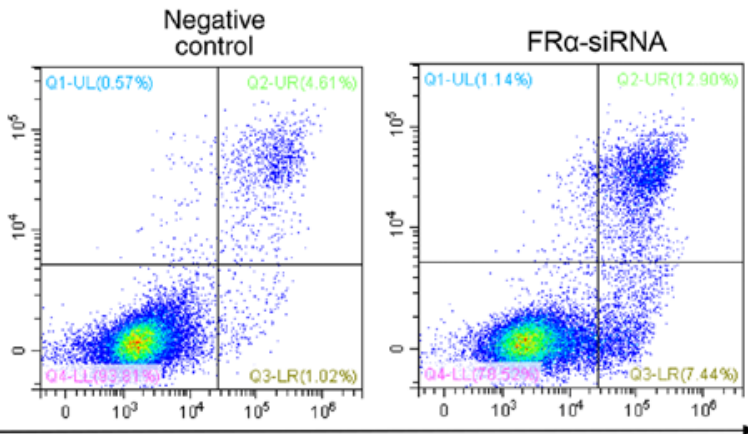

Annexin V-FITC

D

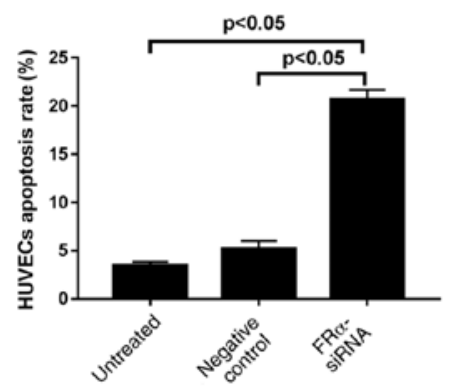

F

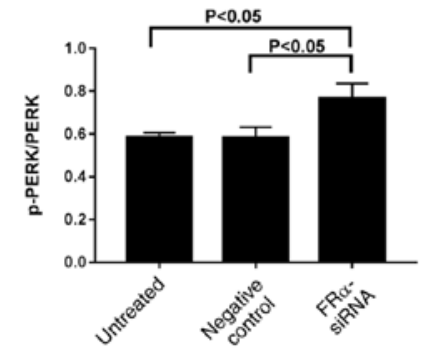

G

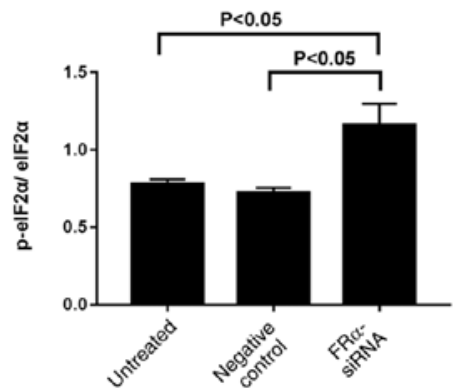

E

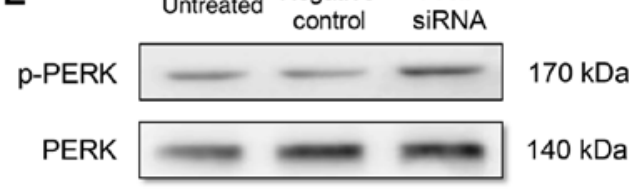

p-elF2a

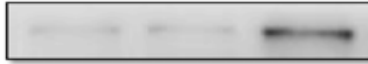

$38 \mathrm{kDa}$

elF2 $\alpha$

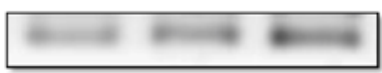

$38 \mathrm{kDa}$

$\beta$-tubulin

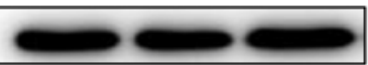

$55 \mathrm{kDa}$

H

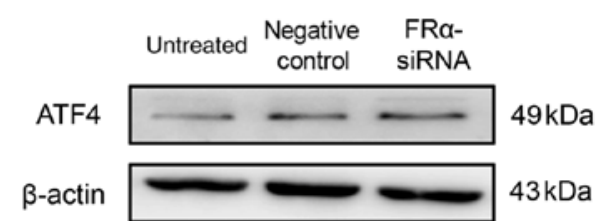

I

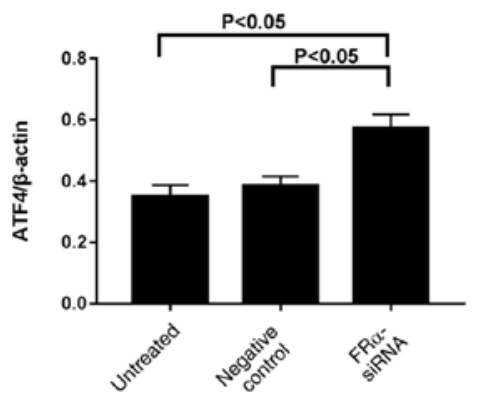

Figure 3. Effect of hFR $\alpha$ inhibition on HUVECs apoptosis and PERK activation. (A) Representative western blot of FR $\alpha$ following knockdown of FR $\alpha$. (B) Semi-quantification of FR $\alpha / \beta$-tubulin. (C) Flow cytometric analysis of HUVECs stained with Annexin V/PI. (D) Quantitative analysis of percentage of apoptotic cells based on flow cytometry of Annexin V/PI double-staining. (E) Representative western blot of PERK, p-PERK, eIF2 $\alpha$ and p-eIF2 $\alpha$. (F) Semi-quantification of p-PERK/PERK. (G) Semi-quantification of p-eIF2 $\alpha /$ IF2 $\alpha$. (H) Representative western blot of ATF4 and (I) semi-quantification of ATF4/ $\beta$-actin. Data are presented as mean \pm standard error. (B, D, F and G) Statistical analysis was conducted by one-way ANOVA followed by Tukey's multiple comparison post hoc test. (I) Statistical analysis was conducted by the Kruskal-Wallis test followed by Dunn-Bonferroni post-hoc test between each group. hFR $\alpha$, human folate receptor $\alpha$; HUVECs, human umbilical vein endothelial cells; PERK, protein kinase RNA-like endoplasmic reticulum kinase; p, phosphorylated; siRNA, small interfering RNA; ATF4, activating transcription factor 4; FITC, fluorescein isothiocyanate; PI, propidium iodide; eIF2 $\alpha$, eukaryotic translation initiation factor $2 \alpha$. 
A

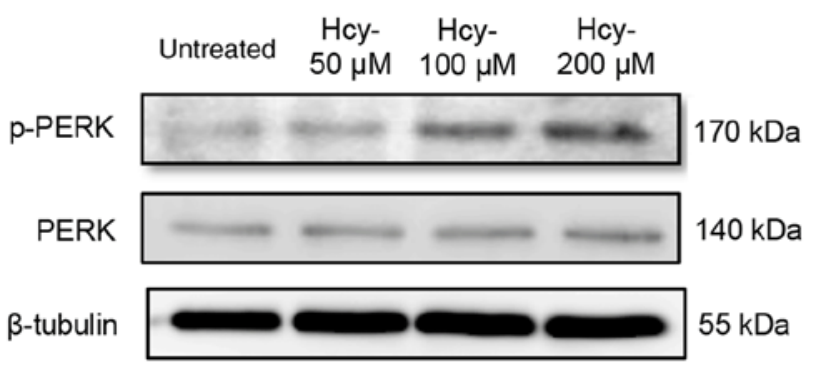

C

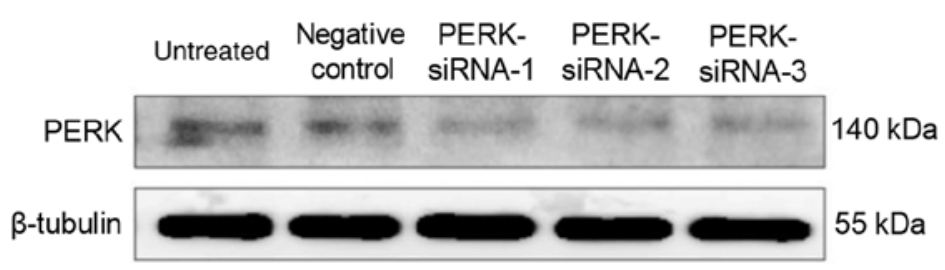

E
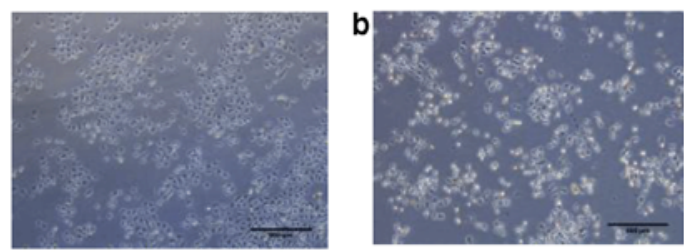

C

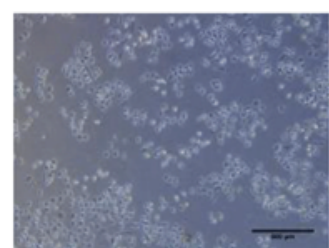

B

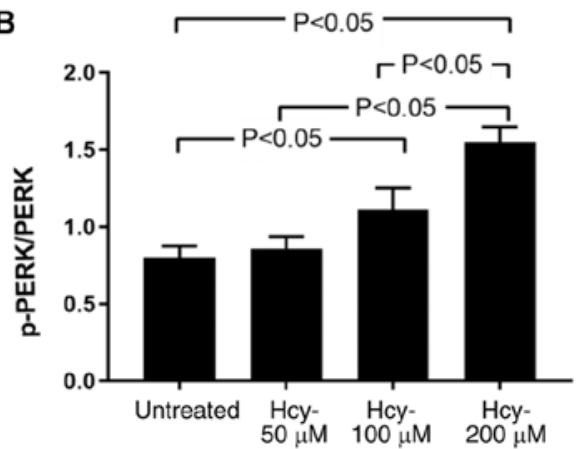

D
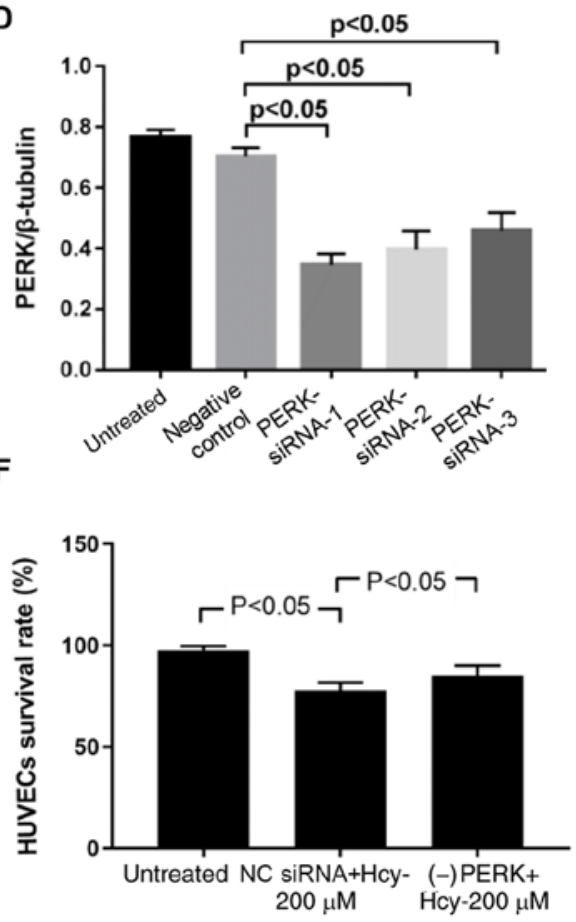

Figure 4. Effect of Hcy treatment on PERK activation, and PERK knockdown on viability of HUVECs. (A) Representative western blot of PERK and (B) semi-quantification of p-PERK/PERK. (C) Representative western blot of PERK following knockdown of PERK. (D) Semi-quantification of PERK/ $\beta$-tubulin. (E) Representative images (magnification, x50) of HUVECs in the (a) untreated, (b) $200 \mu \mathrm{M}$ Hcy + negative control siRNA and (c) $200 \mu \mathrm{M}$ Hcy + PERK siRNA treated cells. (F) Cell Counting Kit-8 assays of cell viability. Data are presented as mean \pm standard error. Statistical analysis was conducted by one-way ANOVA followed by Tukey's multiple comparison post hoc test. HUVECs, human umbilical vein endothelial cells; Hcy, homocysteine; PERK, protein kinase RNA-like endoplasmic reticulum kinase; $p$, phosphorylated; siRNA, small interfering RNA.

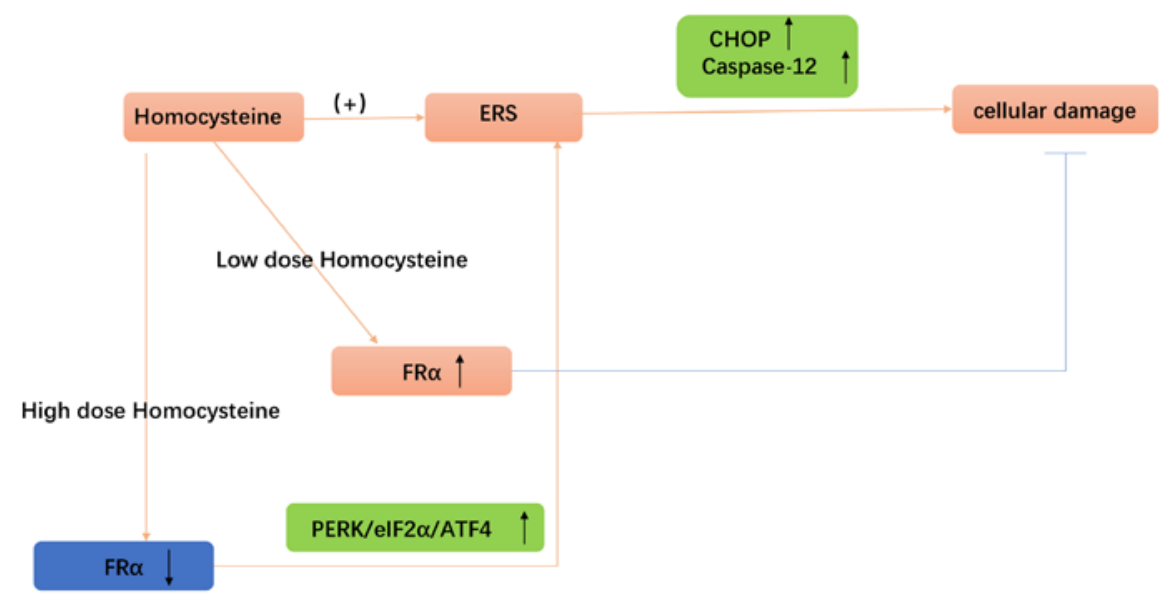

Figure 5. Schematic illustration of the proposed model for hFR $\alpha$ inhibited homocysteine-induced cell injury in HUVECs. ERS, endoplasmic reticulum stress; CHOP, C/EBP homologous protein; PERK, protein kinase RNA-like endoplasmic reticulum kinase; FR $\alpha$, folate receptors $\alpha$; ATF4, activating transcription factor 4; eIF2 $\alpha$, eukaryotic translation initiation factor $2 \alpha$. 
was therefore chosen for further experiments. As shown in Fig. 4E and F, PERK siRNA transfection ameliorated Hcy-induced morphological changes and decreased HUVECs viability $(\mathrm{P}<0.05)$.

\section{Discussion}

Previous studies have demonstrated the detrimental effect of Hcy on HUVECs, which was significantly altered by the addition of folic acid $(8,21,22)$. The present study showed that $\mathrm{hFR} \alpha$ expression was regulated by Hcy and depletion of hFR $\alpha$ mimicked Hcy-induced ER stress and cell injury in HUVECs. The principle findings of the present study were as follows: i) Hcy dose-dependently decreased the expression of $h F R \alpha$ in HUVECs and ii) inhibition of $h F R \alpha$ expression resulted in increased apoptosis and activation of the PERK signaling pathway of ER stress. Collectively, the present study highlighted the critical role of hFR $\alpha$ in protecting against Hcy-induced endothelial injury. The schematic illustration of the proposed model is presented in Fig. 5.

The present study investigated the acute toxicity of mild-to-moderate doses of Hcy in HUVECs, because mild and moderate HHcy is more common in the general population (9). The dose of Hcy used in this study is a similar concentration to what is used in HUVECs in previous reports $(8,23,24)$. Furthermore, 2,000 $\mu \mathrm{M}$ Hcy was reported as a moderate HHcy concentration (8). Therefore, the doses of Hcy used in this study should be considered as low-to-moderate concentrations. Previous studies have demonstrated that mild and moderate HHcy induced apoptosis and injury in HUVECs in a dose-dependent manner $(8,23,24)$. Consistently, this study found that Hcy induced morphological injury and reduced viability of HUVECs, particularly at higher doses. In addition, a previous clinical study reported that in patients at risk for atherosclerosis, $17 \%$ of men with higher Hcy concentrations $(21.27 \pm 5.37 \mu \mathrm{M})$ died during follow up (25). However, the finding in the present study that there were no significant morphological changes to the endothelial cells following treatment with $50 \mu \mathrm{M}$ Hcy is inconsistent with this report (25). This discrepancy might be caused by the different experimental conditions, such as different experimental subject (HUVEC cells vs. a human male), or different study duration ( $24 \mathrm{~h}$ vs. 5 years).

It has been reported that Hcy treatment may reduce HUVEC folate levels and folate supplements can decrease Hcy levels and improve cell viability (21). Since hFRs play a key role in cell folate uptake, it is important to investigate whether Hcy modulates hFR levels in HUVECs. The present study found that hFRs, mainly hFR $\alpha$, exist in HUVECs. Moreover, low-dose Hcy treatment induced an increase in hFR $\alpha$ mRNA and protein levels. Notably, higher dose Hcy induced a decrease in $\mathrm{hFR} \alpha$ expression. To the best of our knowledge, this is the first study to demonstrate that $\mathrm{hFR} \alpha$ exists in HUVECs and can be regulated by Hcy treatment. It has been suggested that intracellular Hcy can stimulate the mRNA expression of FRs, which may represent a mechanism underlying the effect of low-dose Hcy on hFR $\alpha$ levels (26). However, the present study did not allow us to elucidate the precise mechanism of Hcy in the regulation of hFRs. Further study is needed to determine the precise mechanism of Hcy-induced hFR $\alpha$ expression.
It has been reported that ER stress induced by Hcy is one of the main mechanisms for endothelial injury $(8,20,22)$. ER stress is initiated by the accumulation of unfolded proteins, and chronic or severe ER stress in endothelial cells can result in cell death (27). Activation of the PERK pathway, represented by p-PERK expression, is the most definitive marker of ER stress. It has been demonstrated that Hcy can activate the PERK pathway $(8,24)$. However, few studies have investigated the role of $\mathrm{hFR} \alpha$ in ER stress $(8,20)$. This study demonstrated that $\mathrm{hFR} \alpha$ inhibition was associated with PERK signal pathway activation. Additionally, $\mathrm{hFR} \alpha$ downregulation was also associated with an increased apoptotic rate in HUVECs. In agreement with previous studies $(8,20)$, these results showed that PERK was strongly involved in Hcy-mediated HUVEC injury, since depletion of PERK significantly reduced Hcy-induced cell injury. Considering these results, it is possible that $\mathrm{hFR} \alpha$ may serve a protective role against Hcy-induced ER stress and cell injury in HUVECs.

In conclusion, this study revealed that $\mathrm{hFR} \alpha$ was present in HUVECs, and may negatively regulate ER stress, apoptosis and reduced cell viability induced by Hcy treatment in HUVECs. The present results indicated that $\mathrm{hFR} \alpha$ may be an important target for Hcy treatment.

\section{Acknowledgements}

Not applicable.

\section{Funding}

This study was supported by the National Natural Science Foundation of China (grant no. 81560079), Major Projects of the Jiangxi Province Natural Science Foundation of China (grant no. 20152ACB20022) and the Education Department of Jiangxi Province (grant no. GJJ150265).

\section{Availability of data and materials}

The datasets used and/or analyzed during the present study are available from the corresponding author on reasonable request.

\section{Authors' contributions}

The work presented here was carried out in collaboration between each author. JC, CC, HH, LQW and XH performed the experiments. JC, CC and PL wrote the main manuscript. HZ and YJY statistically analyzed the data. PL and JC designed the experiments. All authors read and approved the final manuscript.

\section{Ethics approval and consent to participate}

Not applicable.

\section{Patient consent for publication}

Not applicable.

\section{Competing interests}

The authors declare that they have no competing interests. 


\section{References}

1. Han L, Wu Q, Wang C, Hao Y, Zhao J, Zhang L, Fan R, Liu Y, Li R, Chen Z, et al: Homocysteine, ischemic stroke, and coronary heart disease in hypertensive patients: a population-based, prospective cohort study. Stroke 46: 1777-1786, 2015.

2. Bonetti PO, Lerman LO and Lerman A: Endothelial dysfunction: A marker of atherosclerotic risk. Arterioscler Thromb Vasc Biol 23: 168-175, 2003.

3. Vita JA: Endothelial function. Circulation 124: e906-e912, 2011.

4. Stamler JS, Osborne JA, Jaraki O, Rabbani LE, Mullins M, Singel D and Loscalzo J: Adverse vascular effects of homocysteine are modulated by endothelium-derived relaxing factor and related oxides of nitrogen. J Clin Invest 91: 308-318, 1993.

5. Woo KS, Chook P, Lolin YI, Cheung AS, Chan LT, Sun YY, Sanderson JE, MetreweliC and Celermajer DS: Hyperhomocyst(e) inemia is a risk factor for arterial endothelial dysfunction in humans. Circulation 96: 2542-2544, 1997.

6. Cimellaro A, Perticone M, Fiorentino TV, Sciacqua A and Hribal ML: Role of endoplasmic reticulum stress in endothelial dysfunction. Nutr Metab Cardiovasc Dis 26: 863-871, 2016.

7. Wang XC, Sun WT, Yu CM, Pun SH, Underwood MJ, He GW and Yang Q: ER stress mediates homocysteine-induced endothelial dysfunction: Modulation of IKCa and SKCa channels. Atherosclerosis 242: 191-198, 2015.

8. Zhang Z, Wei C, Zhou Y, Yan T, Wang Z, Li W and Zhao L: Homocysteine induces apoptosis of human umbilical vein endothelial cells via mitochondrial dysfunction and endoplasmic reticulum stress. Oxid Med Cell Longev 2017: 5736506, 2017.

9. De Bree A, Verschuren WM, Kromhout D, Kluijtmans LA and Blom HJ: Homocysteine determinants and the evidence to what extent homocysteine determines the risk of coronary heart disease. Pharmacol Rev 54: 599-618, 2002.

10. Casas JP, Bautista LE, Smeeth L, Sharma P and Hingorani AD: Homocysteine and stroke: Evidence on a causal link from mendelian randomisation. Lancet 365: 224-232, 2005.

11. Huo Y, Li J, Qin X, Huang Y, Wang X, Gottesman RF, Tang G, Wang B, Chen D, He M, et al; CSPPT Investigators: Efficacy of folic acid therapy in primary prevention of stroke among adults with hypertension in China: The CSPPT randomized clinical trial. JAMA 313: 1325-1335, 2015.

12. Klerk M, Verhoef P, Clarke R, Blom HJ, Kok FJ and Schouten EG; MTHFR Studies Collaboration Group: MTHFR 677C-->T polymorphism and risk of coronary heart disease: A meta-analysis. JAMA 288: 2023-2031, 2002.

13. Mark SD, Wang W, Fraumeni JF Jr, Li JY, Taylor PR, Wang GQ, Guo W, Dawsey SM, Li B and Blot WJ: Lowered risks of hypertension and cerebrovascular disease after vitamin/mineral supplementation: The Linxian Nutrition Intervention Trial. Am J Epidemiol 143: 658-664, 1996.
14. Chen C, Ke J, Zhou XE, Yi W, Brunzelle JS, Li J, Yong EL, $\mathrm{Xu} \mathrm{HE}$ and Melcher K: Structural basis for molecular recognition of folic acid by folate receptors. Nature 500: 486-489, 2013.

15. Parker N, Turk MJ, Westrick E, Lewis JD, Low PS and Leamon CP: Folate receptor expression in carcinomas and normal tissues determined by a quantitative radioligand binding assay. Anal Biochem 338: 284-293, 2005.

16. Weitman SD, Weinberg AG, Coney LR, Zurawski VR, Jennings DS and Kamen BA: Cellular localization of the folate receptor: Potential role in drug toxicity and folate homeostasis. Cancer Res 52: 6708-6711, 1992.

17. Hou Z and Matherly LH: Biology of the major facilitative folate transporters SLC19A1 and SLC46A1. Curr Top Membr 73: 175-204, 2014.

18. Qiu A, Jansen M, Sakaris A, Min SH, Chattopadhyay S, Tsai E, Sandoval C, Zhao R, Akabas MH and Goldman ID: Identification of an intestinal folate transporter and the molecular basis for hereditary folate malabsorption. Cell 127: 917-928, 2006.

19. Schmittgen TD and Livak KJ: Analyzing real-time PCR data by the comparative C(T) method. Nat Protoc 3: 1101-1108, 2008.

20. Harding HP, Zhang Y, Bertolotti A, Zeng H and Ron D: Perk is essential for translational regulation and cell survival during the unfolded protein response. Mol Cell 5: 897-904, 2000.

21. Cui S, Li W, Wang P, Lv X, Gao Y and Huang G: Folic acid inhibits homocysteine-induced cell apoptosis in human umbilical vein endothelial cells. Mol Cell Biochem 444: 77-86, 2018.

22. Wu X, Zhang L, Miao Y, Yang J, Wang X, Wang CC, Feng J and Wang L: Homocysteine causes vascular endothelial dysfunction by disrupting endoplasmic reticulum redox homeostasis. Redox Biol 20: 46-59, 2019.

23. Bao XM, Wu CF and Lu GP: Atorvastatin attenuates homocysteine-induced apoptosis in human umbilical vein endothelial cells via inhibiting NADPH oxidase-related oxidative stress-triggered p38MAPK signaling. Acta Pharmacol Sin 30: 1392-1398, 2009.

24. Zhu L, Jia F, Wei J, Yu Y, Yu T, Wang Y, Sun J and Luo G: Salidroside protects against homocysteine-induced injury in human umbilical vein endothelial cells via the regulation of endoplasmic reticulum stress. Cardiovasc Ther 35: 33-39, 2017.

25. Blum A, Hijazi I, Eizenberg MM and Blum N: Homocysteine (Hcy) follow-up study. Clin Invest Med 30: 21-25, 2007.

26. Perna AF, Lanza D, Sepe I, Conzo G, Altucci L and Ingrosso D: Altered folate receptor 2 expression in uraemic patients on haemodialysis: Implications for folate resistance. Nephrol Dial Transplant 28: 1214-1224, 2013.

27. Hotamisligil GS: Endoplasmic reticulum stress and the inflammatory basis of metabolic disease. Cell 140: 900-917, 2010. 